\title{
ANÁLISE DE ATIVIDADE DE ÁGUA E UMIDADE NA QUALIDADE DO MEL PRODUZIDO EM COMUNIDADES DA RESERVA EXTRATIVISTA TAPAJÓS- ARAPIUNS, SANTARÉM, PARÁ
}

\author{
Yasmin dos Santos Picanço'; Sullyvan Silva Oliveira²; Monique Almeida³; Fabrizia Sayuri Otani ${ }^{4}$; Eliriane \\ Jamas Pereira ${ }^{5}$; Graciene Conceição dos Santos ${ }^{6}$. \\ ${ }^{1}$ Universidade Federal do Oeste do Pará, Santarém, Pará, Brasil, yasmindossantospicanco@gmail.com \\ ${ }^{2}$ Universidade Federal do Oeste do Pará, Santarém, Pará, Brasil, sullyvanoliveira23@gmail.com \\ ${ }^{3}$ Universidade Federal do Oeste do Pará, Santarém, Pará, Brasil, moniquealmeida317@gmail.com \\ ${ }^{4}$ Universidade Federal do Oeste do Pará, Santarém, Pará, Brasil, fabrizia_otani@yahoo.com.br \\ ${ }^{5}$ Faculdades Integradas de Bauru, Bauru, São Paulo, Brasil, eliriane@gmail.com \\ ${ }^{6}$ Universidade Federal do Oeste do Pará, Santarém, Pará, Brasil, gracienecsantos@yahoo.com.br
}

RESUMO: O objetivo do presente trabalho foi avaliar a atividade de água (Aw) e umidade em méis de Scaptotrigona sp. produzidos em três comunidades da Reserva extrativista Tapajós-Arapiuns, Santarém/PA. Foi utilizado 9 amostras de méis, sendo 2 da comunidade de Solimões, 4 da comunidade de Carão e 3 da comunidade de Anã. As amostras foram armazenadas e refrigeradas até as análises em triplicata e utilizou-se o software SISVAR para a análise de variância pelo teste de Tukey $(p<0,05)$. Os resultados obtidos para atividade de água variaram de 0,68 a 0,77, sendo o mínimo correspondente a comunidade de Anã e o máximo a comunidade de Solimões. O limite para atividade de água no mel de abelhas sem ferrão está entre 0,52 e 0,80, logo, a Aw das amostras do presente trabalho estão dentro do limite permitido para que não haja a deterioração e perda de qualidade do mel. Com relação a umidade, as médias estão entre 24,37 e 33,86, sendo a maior média de Solimões e a menor de Anã. Apesar de não haver um parâmetro oficial para méis de meliponíneos, alguns autores recomendam para esses méis umidade máxima de 35\%. Logo, os teores de umidade obtidos estão dentro do recomendado, porém necessita de mais pesquisas a fim de criar uma legislação específica para méis de abelhas sem ferrão, já que este possui características distintas.

PALAVRA-CHAVE: Deterioração, Microrganismos, Prateleira.

\section{ANALYSIS OF WATER ACTIVITY AND HUMIDITY IN HONEY QUALITY OF PRODUCED IN COMMUNITIES OF THE TAPAJÓS EXTRATIVIST RESERVE- ARAPIUNS, SANTARÉM, PARÁ}

ABSTRACT: This work aimed to evaluate the water activity (Aw) and humidity in honey of Scaptotrigona sp. produced in three communities of the Tapajós - Arapiuns Extractive Reserve, Santarém / PA. It was used 9 samples of honeys, being 2 of the community of Solimões, 4 of the community of Carão and 3 of the community of Anã. The samples were stored and refrigerated until the analyzes in triplicate and the SISVAR software was used for analysis of variance by Tukey's test $(p<0.05)$. The results obtained for water activity varied from 0.68 to 0.77 , the minimum corresponding to the community of Anã and the 
maximum the community of Solimões. The limit for water activity in honey from stingless bees is between 0.52 and 0.80 , so the Aw of the samples of the present work are within the limit allowed so that there is no deterioration and loss of honey quality. In relation to humidity, the averages are between 24,37 and 33,86, being the highest average of Solimões and the lowest of Dwarf. Although there is no official parameter for meliponine honeys, some authors recommend maximum moisture of $35 \%$ for these honeys. Therefore, the moisture content obtained is within the recommended range, but needs further research to create specific legislation for bee honeys without sting, since it has different characteristics.

KEYWORD: Deterioration, Microorganisms, Shelf.

\section{ANÁLISIS DE ACTIVIDAD DE AGUA Y HUMEDAD EN LA CALIDAD DEL MEL PRODUCIDO EN COMUNIDADES DE LA RESERVA EXTRATIVISTA TAPAJÓS - ARAPIUNS, SANTARÉM, PARÁ}

RESUMEN: Este trabajo objetivó evaluar la actividad de agua (Aw) y humedad en las mentes de Scaptotrigona sp. producidos en tres comunidades de la Reserva extractivista Tapajós - Arapiuns, Santarém / PA. Se utilizaron 9 muestras de meles, siendo 2 de la comunidad de Solimões, 4 de la comunidad de Carón y 3 de la comunidad de Enan. Las muestras fueron almacenadas y refrigeradas hasta los análisis en triplicada y se utilizó el software SISVAR para el análisis de varianza por el test de Tukey $(p<0,05)$. Los resultados obtenidos para la actividad de agua variaron de 0,68 a 0,77, siendo el mínimo correspondiente a la comunidad de Enan y el máximo a la comunidad de Solimões. El límite para actividad de agua en la miel de abejas sin aguijón está entre 0,52 y 0,80, luego, la Aw de las muestras del presente trabajo están dentro del límite permitido para que no haya el deterioro y pérdida de calidad de la miel. Con respecto a la humedad, las medias están entre 24,37 y 33,86, siendo la mayor media de Solimões y la menor de Enan. A pesar de que no hay un parámetro oficial para los melones de melipón, algunos autores recomiendan para estés mieles humedad máxima del 35\%. Por lo tanto, los contenidos de humedad obtenidos están dentro de lo recomendado, pero necesita más investigaciones para crear una legislación específica para las muelas de abejas sin aguijón, ya que éste posee características distintas.

PALABRAS CLAVE: Deterioro, Estante, Microorganismos.

\section{INTRODUÇÃO}

As abelhas sem ferrão ou nativas, são espécies de abelhas sociais e, de acordo com Camargo e Pedro (2007), sua maior diversidade localiza-se nos trópicos americanos, com aproximadamente 400 espécies descritas. Segundo informações do Instituto Peabiru (2016), o mel dessas 
abelhas apresenta características físicoquímicas distintas daquelas conhecias para o gênero Apis, por exemplo, mais líquido. A qualidade do mel é dependente das características que ele possui, tais como: cor, sabor, aroma, cristalização, umidade, viscosidade, entre outras.

No que diz respeito as propriedades físico-químicas do mel, a umidade e a atividade de água (Aw) são parâmetros importantes a serem considerados. Segundo Felsner e Cano (2011), a umidade é a quantidade de água presente nos alimentos, e no mel afeta propriedades físicas e sensoriais como cor, viscosidade, sabor, peso específico, solubilidade, determinando, desta forma, seu valor comercial. A atividade de água, por sua vez, é a água disponível do alimento que vai reagir com os microorganismos. Então, quanto mais elevada a Aw, maior a predisposição ao desenvolvimento de fungos, leveduras e bactérias.

Ter conhecimento dessas características é imprescindível, pois produtos com elevado teor de açúcar em geral apresentam Aw baixa, o que os caracteriza como microbiologicamente estáveis, porém tendem a absorver umidade. O mel, por ser um produto composto de açúcares (65 a 70\%), apresenta alta higroscopicidade, isto é, pode absorver água, como também ceder (eliminar água) (MERABET et al., 2011).

Apesar de sua importância, a atividade de água não é considerada parâmetro a ser estudado na legislação para méis, no entanto o conhecimento desses valores ajuda a determinar a vida de prateleira, a escolher melhor os tipos de embalagens e as condições de armazenamento (CORREIA-OLIVEIRA et al., 2008). Logo, é importante saber mais sobre a Aw e a umidade no mel e, com isso, proporcionar um produto em boas condições ao consumidor, livre de fatores que diminuem a sua qualidade.

Segundo Yamamoto et al. (2007), há esforços para alavancar o desenvolvimento da meliponicultura no Brasil. Contudo, é necessário estabelecerem-se requisitos de identidade padrão que garantam a segurança de consumo e para que os órgãos fiscalizadores tenham base referencial para sua inspeção, permitindo a comercialização oficial desse produto.

Partindo desse pressuposto, o objetivo do presente trabalho foi avaliar a atividade de água (Aw) e umidade em amostras de 
méis de Scaptotrigona sp. produzidos em três comunidades localizadas na reserva extrativista tapajós - Arapiuns, Santarém/PA.

\section{MATERIAL E MÉTODOS}

Foram utilizadas 9 amostras de méis de abelhas sociais sem ferrão, sendo duas amostras de Scaptotrigona sp., coletados na comunidade de Solimões, quatro amostras de Scaptotrigona sp., coletados na comunidade de Carão e sendo três amostras de Scaptotrigona sp., coletados na comunidade de Anã. As amostras foram coletadas no mês de setembro de 2016.

Essas comunidades localizam-se dentro da área de Reserva Extrativista TapajósArapiuns (Resex). A Resex TapajósArapiuns encontra-se nos municípios de Santarém e Aveiro, ao oeste do Estado do Pará. Dos 647.610 hectares de sua área, cerca de 34\% (194.283 ha) estão localizados no município de Aveiro/PA. E 66\% (453.327 ha), no município de Santarém/PA.

A oeste, a RESEX tem seus limites marcados pelos rios Arapiuns, Maró e Mentai; pelas glebas Mamuru e Nova Olinda e pelo Projeto de Assentamento
Agroextrativista (PAE) Lago Grande. Já o limite Leste é dado pelo rio Tapajós. O nome da Unidade de Conservação se deve a sua localização entre os rios Tapajós e Arapiuns. Na parte Norte da RESEX, esses dois rios se encontram na frente da cidade de Santarém (Figura 1).

As amostras de méis foram obtidas diretamente dos potes nas caixas racionais, o méis foram colhidas por meio de sucção com seringas descartáveis, armazenadas em potes de plástico estéril de polietileno, de fechamento hermético e mantidas sob refrigeração de aproximadamente 5,0 드. até a realização das análises - em triplicata - que foram conduzidas no Laboratório de Tecnologia de Produtos de Origem Animal na Universidade Federal Oeste do Pará e no Laboratório de Análises de Alimento da UNESP, Campus de Botucatu.

Para a avaliação da atividade de água das amostras (Aw -activity water), foi utilizado o aparelho AquaLab, que utiliza a técnica de determinação do ponto de orvalho para medir a atividade de água de um produto. Sendo colocado aproximadamente um grama de amostra no aparelho. 
Figura 1. Localização das comunidades de Solimões, Carão e Anã na Resex Tapajós/Arapiuns.

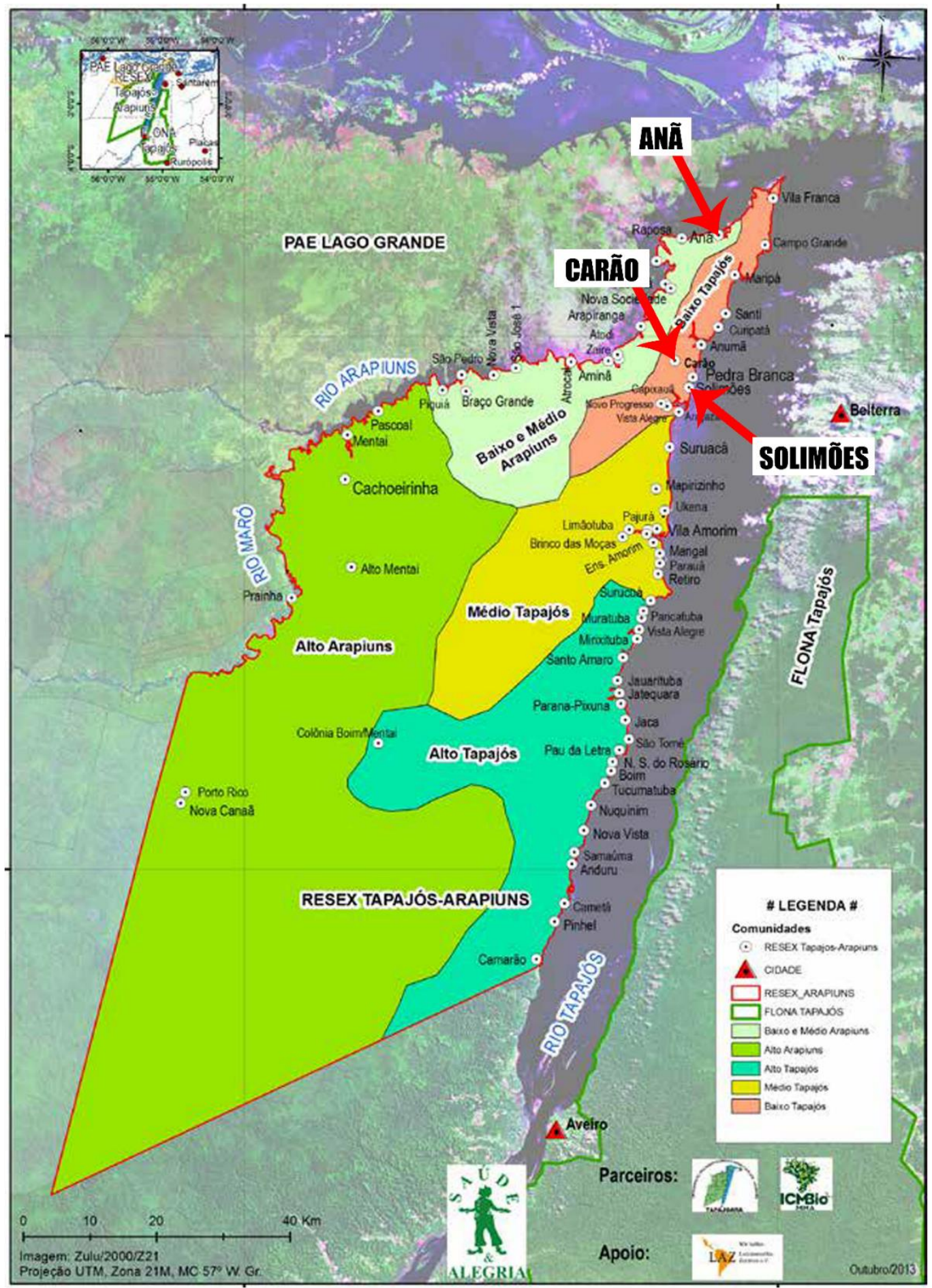

Fonte: Almanaque da Resex (2013). 
Para análise de umidade foi pesada $5 \mathrm{~g}$ de cada amostra em uma cápsula de porcelana, previamente tarada. A desidratação foi efetuada numa estufa a $110 \stackrel{\circ}{ } \mathrm{C}$, por cinco horas, submetido ao resfriamento no dessecador e, por último realizou-se a pesagem.

Utilizou-se o software SISVAR para a análise de variância (ANOVA) pelo teste de Tukey, com o objetivo de analisar diferenças significativas $(p<0,05)$ entre as médias aritméticas dos grupos.

\section{RESULTADOS E DISCUSSÃO}

O mel das abelhas sem ferrão costuma apresentar mais água em sua composição comparado ao mel de Apis melífera. Camargo et al. (2017) considera que o limite para atividade de água no mel de abelhas sem ferrão está entre 0,52 e 0,80, logo, a atividade de água das amostras do presente trabalho estão dentro do limite permitido para que não haja a deterioração desse mel e a perda de sua qualidade (Tabela 1).

Tabela 1. Valores da atividade de água (AW) no mel das abelhas Canudo (Scaptotrigona sp.) nas comunidades de Solimões, Carão e Anã.

\begin{tabular}{|c|c|c|}
\hline Comunidade & Amostra & Atividade de água (AW) \\
\hline \multirow[t]{2}{*}{ Solimões } & Canudo (Scaptotrigona sp) 1 & $0,73 \mathrm{e}$ \\
\hline & Canudo (Scaptotrigona sp) 2 & $0,77 \mathrm{a}$ \\
\hline \multirow[t]{4}{*}{ Carão } & Canudo (Scaptotrigona sp) 3 & $0,75 c$ \\
\hline & Canudo (Scaptotrigona sp) 4 & $0,72 \mathrm{f}$ \\
\hline & Canudo (Scaptotrigona sp) 5 & $0,75 c$ \\
\hline & Canudo (Scaptotrigona sp) 6 & $0,76 b$ \\
\hline \multirow[t]{3}{*}{ Anã } & Canudo (Scaptotrigona sp) 7 & $0,68 \mathrm{i}$ \\
\hline & Canudo (Scaptotrigona sp) 8 & $0,71 \mathrm{~g}$ \\
\hline & Canudo (Scaptotrigona sp) 9 & $0,68 \mathrm{~h}$ \\
\hline CV (\%) & 0,46 & \\
\hline
\end{tabular}

Medidas seguidas de letras iguais não diferem pelo teste de Turkey $(P<0,05 \%)$.

Valores próximos dos resultados no presente trabalho foram encontrados por Borges (2012) para méis de espécies do gênero Scaptotrigona sp onde obteve-se uma variação entre 0,72 e 0,79aw. Já Barbosa (2013) avaliou a atividade de água no mel de abelhas Apis melifera, e obteve valores entre 0,58 e 0,60. Isso demonstra a diferença da atividade de água para méis de Apis comparada a méis de Meliponas.

Valores para atividade de água acima dos encontrados no presente trabalho 
foram obtidos por Souza et al. (2009). O autor analisou Aw em méis de Scaptotrigona e o valor máximo encontrado foi de 0,837aw.

Como se sabe, a atividade de água e a umidade são parâmetros que influenciam em sua vida de prateleira e qualidade. Como as qualidades sanitárias, organolépticas e nutricionais são instrumentos fundamentais para se obter vantagens no mercado, é necessário empregar técnicas que garantam maior vida de prateleira desse produto (OLIVEIRA et al., 2013). Em vista a conservação desse produto, existem alguns tratamentos, como o térmico, podem ser utilizados, desde que mantenham os padrões de identidade e qualidade, destacando-se a importância da realização de estudos com análises físico-químicas do mel.

O mel sofre modificações de acordo com o ambiente em que é produzido, com isso é influenciado pela qualidade, quantidade e variedade das plantas que florescem e produzem néctar em uma determinada região (GOMES; SANTOS, 2016). Essa é a principal hipótese que explica os teores de umidade no presente trabalho (Tabela 2), tenho em vista que os maiores valores foram da comunidade de Solimões localizada mais próximo ao rio.

Segundo Villas-Bôas et al. (2012), o mel de meliponíneos apresenta percentuais de umidade maiores comparado aos valores máximos sugeridos para o mel de Apis melifera, limite máximo de 20\%, ideal de 17 a 18\%. Embora não haja um parâmetro oficial para esses méis, alguns autores recomendam umidade máxima de 35\%. Logo, os teores de umidade obtidos no presente trabalho estão dentro dos limites encontrados na literatura, porém são necessitas mais pesquisas para que se crie legislação específica para méis de abelhas sem ferrão, já que este possui características particulares.

Fugii et al. (2009), analisaram o teor de umidade de méis de várias espécies de abelhas, dentre eles o mel de Scaptotrigona sp e obtiveram um teor de umidade de 27,7 e 28,3, respectivamente. Lira et al. (2014), constatou em sua pesquisa com méis de Scaptotrigona $s p$, coletados no municípo Belterra/Pa, valores médios para umidade de $27,15 \%$. Os resultados desses autores se diferem dos obtidos no trabalho onde a umidade máxima observada foi de 33,86\%. 
Tabela 2. Médias dos valores de Atividade de água (AW) e Umidade.

\begin{tabular}{lcc}
\hline Amostra & AW & Umidade \\
\hline Solimões (Scaptotrigona sp) & $0,75 \mathrm{a}$ & $33,86 \mathrm{a}$ \\
Carão (Scaptotrigona $s p)$ & $0,74 \mathrm{a}$ & $26,50 \mathrm{~b}$ \\
Anã (Scaptotrigona $s p)$ & $0,69 \mathrm{~b}$ & $24,37 \mathrm{~b}$ \\
\hline CV (\%) & 2,65 & 6,49 \\
\hline
\end{tabular}

Medidas seguidas de letras iguais não diferem pelo teste de Turkey $(P<0,05 \%)$

O teor de umidade é influenciado por vários fatores, dentre eles a espécie, a região em questão e como essas amostram foram conservadas. Prova disso são os resultados do trabalho de Sousa et al. (2013) feito em Seridó/RN, onde foi analisado o teor de umidade no mel de espécies meliponíneas, dentre elas a Melipona scutellaris L. com valores médios de $35,4 \%$. Comparando-se os resultados deste autor com o do presente trabalho, é possível visualizar como se dá a diferença de umidade entre as espécies.

Em seu trabalho com méis de abelhas sem ferrão, Chaves et al. (2012) identificou valor médio para umidade de 30,93\%, embora esse valor esteja acima do permitido pela legislação das abelhas do gênero Apis, demonstra uma característica básica dos méis de abelhas sem ferrão, que é a alta higroscopicidade (facilidade no ganho e na perda de água). Um ambiente com alta umidade relativa induz a trocas em sua composição, alterando a Aw e, consequentemente, favorecendo a deterioração, por isso deve-se evitar a coleta de méis em dias chuvosos, onde poderia aumentar a umidade.

Saber a correlação existente entre umidade e atividade de água é de grande relevância para certificar a qualidade do produto. Em uma linha comparativa dos resultados obtidos no trabalho, os valores mais altos de atividade de água correspondem aos valores mais elevados de umidade. Isso testifica a influência da umidade sobre a Aw.

É importante analisar a quantidade de água nos méis de abelhas sem ferrão para que sua qualidade seja mantida, além de determinar a vida de prateleira desse produto favorecendo o produtor e os consumidores. No entanto, são necessários mais estudos sobre as propriedades do mel para que se constitua uma legislação específica para 
méis de abelhas sem ferrão estabelecendo parâmetros de qualidade ao produto.

\section{CONCLUSÃO}

Com relação aos teores obtidos de Aw obteve-se valores entre 0,68 a 0,77 , sendo o maior valor encontrado correspondente a Solimões e o menor correspondente a Anã. As médias de umidade encontradas estão entre 24,37 e 33,86 , sendo a maior proveniente de Solimões e a menor da comunidade de Anã. Os resultados de Aw e umidade encontram-se dentro dos dados observados na literatura.

\section{REFERÊNCIAS}

BARBOSA, J. S. Avaliação físico-química de méis comercializados nas feiras de Imperatriz-MA. Imperatriz-MA, 2013. Monografia (Graduação em Engenharia de Alimentos do Campus de Imperatriz) - Universidade Federal do Maranhão, Imperatriz/MA, 2013.

BORGES, J. A. C. P. Avaliação microbiológica, atividade de água $\mathrm{e}$ umidade em méis de espécies de abelhas sociais sem ferrão (Apidae: meliponinae) de municípios do território de Irecê - BA. Cruz das Almas, BA, 2012. Monografia (Graduação em Ciências Biológicas) Universidade Federal do Recôncavo da Bahia, Cruz das Almas/BA, 2012.
CAMARGO, J. M. F.; PEDRO, S. R. M. Meliponini Lepeletier, 1836. In: MOURE, J. S.; RBAN, D.; MELO, G. A. R. (Orgs.). Catalogue of bees (Hymenoptera, Apoidea) in the Neotropical Region. Curitiba: Sociedade Brasileira de Entomologia, 2007. p. 272-578.

CAMARGO, R. C. R.; OLIVEIRA, K. L.; BERTO, M. I. Mel de abelhas sem ferrão: proposta de regulamentação. Brazilian Journal of Food Technology, Campinas, v. 20, 2017.

CHAVES, A. F. A.; GOMES, J. E. H.; COSTA, A. J. S. Caracterização físico-química do mel de Melipona fulva Lepeletier, 1836 (Himenoptera: Apidae: Meliponinae) utilizada na meliponicultura por comunidades tradicionais do entorno da cidade de Macapá-AP. Revista Biota Amazônia. Macapá, v. 2, n. 1, p. 1-9, 2012.

CORREIA-OLIVEIRA, M. E.; FERREIRA, A. F.; PODEROSO, J. C. M.; LESSA, A. C. V.; ARAÚJO, E. D.; CARNELOSSI, M. A. G.; RIBEIRO, G. T. Atividade de água (Aw) em amostras de pólen apícola desidratado e mel do Estado de Sergipe. Revista da Fapese, v. 4, n. 2, p. 27-36, jul./dez. 2008.

FELSNER, M. L.; CANO, C. B. Influência de Variáveis Experimentais na Determinação Refratométrica de Umidade em Mel por Planejamento Fatorial. Revista Ciências Exatas e Naturais, v. 13, n. 1, jan./jun. 2011.

FUJII, I.; RODRIGUES, P. R. M.; FERREIRA, M. N.Caracterização físico-química do mel de guaranazeiro (Paullinia cupana varsorbilis) em Alta Floresta, Mato Grosso. Revista Brasileira de Saúde e Produção Animal, v. 10, n. 3, p. 645-653 jul/set, 2009. 
GOMES, S. J. S.; SANTOS, C. V. Consumo e Mercado do Mel: Um Estudo Bibliográfico. Revista Síntese AEDA, v.01, n. 02 - Jul- Dez - 2016.

INSTITUTO PEABIRU. Criação de abelhas nativas (meliponicultura) pela agricultura familiar da Amazônia Meliponicultura legal, $2^{\circ}$ versão. Campanha para a autorização de manejo simplificado da meliponicultura na Amazônia. Novembro, 2016.

LIRA, A. F.; SOUZA, P. L. M.; LORENZON, M. C. A.; VIANNA, C. A. F. J.; CASTRO, R. N. Estudo Comparativo do Mel de Apis Mellifera com méis de Meliponíneos. Revista Acta Veterinaria Brasilica, v.8, n.3, p.169-178, 2014.

MERABET, L. P. Determinação de atividade de água, teor de umidade e parâmetros microbiológicos em compostos de mel. Revista Brasileira de Economia Doméstica, Viçosa, v. 22, n. 2, p.213-232, 2011.

OLIVEIRA, D. J.; SILVA, D. S. M.; SOUZA, A. V.; JUNIOR, C. A. L.; SODRÉ, G. S.; CARVALHO, C. A. L. Avaliação de métodos de conservação do mel de Melipona quadrifasciata com base no perfil sensorial e aceitabilidade. Revista Magistra, Cruz das Almas-BA, v. 25, n. 1, p. 1-6, jan./mar. 2013.

PENA, F.; HENRIQUES, G. Almanaque da reserva extrativista Tapajós-Arapiuns Projeto Saúde e Alegria, ISBN: 978-8568946-01-5. 2015.

SOUSA, J. M. B.; AQUINO, I. S.; MAGNANI M.; ALBUQUERQUE, J. R.; SANTOS, G. G.; SOUZA, E. L. Aspectos físico-químicos e perfil sensorial de méis de abelhas sem ferrão da região do Seridó, Estado do Rio
Grande do Norte, Brasil. Revista Semina: Ciências Agrárias, Londrina, v. 34, n. 4, p. 1765-1774, jul./ago. 2013.

SOUZA, B. A.; MARCHINI, L. C.; DIAS, C. T. S.; ODA-SOUZA, M.; CARVALHO, C. A. L.; ALVES, R. M. O. Avaliação microbiológica de amostras de mel de trigoníneos (Apidae: Trigonini) do Estado da Bahia. Revista Ciência e Tecnologia de Alimentos, Campinas, v. 29, n. 4, 798-798 802, out./dez. 2009.

VILLAS-BÔAS, J. Manual tecnológico: abelhas sem ferrão. 96 p. (Série Manual Tecnológico). Brasília, DF: Instituto Sociedade, População e Natureza (ISPN), 2012.

YAMAMOTO, D. Y.; AKATSU, I. P.; SOARES, A. E. E. Quantificação da produção do mel de Scaptotrigona aff. depilis (hymenoptera, apidae, apinae) do município de Luiz Antônio, São Paulo, Brasil. Revista Bioscience Journal, Uberlândia, v. 23, Supplement 1, p. 8993, nov. 2007. 